Report no. 03/06

\title{
Optimal Error Estimates for the $h p$-Version Interior Penalty Discontinuous Galerkin Finite Element Method
}

\author{
Emmanuil H. Georgoulis and Endre Süli \\ Oxford University Computing Laboratory \\ Numerical Analysis Group
}

\begin{abstract}
We consider the $h p$-version interior penalty discontinuous Galerkin finite element method ( $h p$-DGFEM) for second-order linear reaction-diffusion equations. To the best of our knowledge, the sharpest known error bounds for the $h p$-DGFEM are due to Riviére, Wheeler and Girault [8] and due to Houston, Schwab and Süli [5] which are optimal with respect to the meshsize $h$ but suboptimal with respect to the polynomial degree $p$ by half an order of $p$. We present improved error bounds in the energy norm, by introducing a new function space framework. More specifically, assuming that the solutions belong element-wise to an augmented Sobolev space, we deduce $h p$-optimal error bounds.
\end{abstract}

Key words and phrases: interior penalty discontinuous Galerkin methods, reaction-diffusion PDEs, $h p$-optimal estimates, augmented Sobolev spaces

Oxford University Computing Laboratory

Numerical Analysis Group

Wolfson Building

Parks Road

Oxford, England OX1 3QD

November, 2003 


\section{Introduction}

In recent years, there has been an increasing interest in a type of non-conforming finite element approximation to solutions of elliptic boundary-value problems, usually referred to as discontinuous Galerkin finite element method (DGFEM). Justifications for the renewed interest in these methods, which date back to the 1970s and the early 1980s [6, 10,1 ], can be found in the very attractive properties they exhibit, such as great flexibility in mesh design (irregular grids are perfectly admissible), the freedom of choosing the elemental polynomial degrees without worrying about the enforcement of any interelement conformity requirements, good local conservation properties of the state variable, and good stability properties near boundary/interior layers or even discontinuities [2]. The first two reasons mentioned above make discontinuous Galerkin methods significant contenders for $h p$-adaptivity, whereas the last two are attractive when convection is the dominant feature of the problem.

To the best of our knowledge, the sharpest known error bounds for the $h p$-version DGFEM ( $h p$-DGFEM) for second-order elliptic PDEs are due to Riviére, Wheeler and Girault [8] and Houston, Schwab and Süli [5]; they are optimal with respect to the meshsize $h$ but are suboptimal with respect to the polynomial degree $p$ by half an order of $p$. This work presents a new functional analytic framework for deriving error bounds for the interior penalty discontinuous Galerkin finite element method proposed in $[1,8$, $5]$, and removes the suboptimality in the polynomial degree $p$ in the bounds previously presented in the literature $[8,5]$.

The paper is organized as follows. We begin by introducing the model problem (Section 2) followed by a discussion on function spaces (Section 3) where, along with the (standard) Hilbertian Sobolev spaces $H^{k}$, a new class of augmented Sobolev spaces is defined. Next, the broken weak formulation (Section 4), the admissible finite element spaces and the $h p$-DGFEM (Section 5) are defined. Also, new approximation error estimates for the $H^{1}$-projection operator in various norms are established in Section 6; these are used in the error analysis presented in Section 7.

\section{Model Problem}

Let $\Omega$ be a bounded open (curvilinear) polygonal domain in $\mathbb{R}^{2}$, and let $\Gamma_{\partial}$ signify the union of its one-dimensional open edges, which are assumed to be sufficiently smooth (in a sense defined rigorously later). We consider the reaction-diffusion equation

$$
\mathcal{L} u \equiv-\nabla \cdot(a \nabla u)+c u=f \quad \text { in } \Omega,
$$

where $f \in L^{2}(\Omega), c \in L^{\infty}(\Omega)$, and $a=\left\{a_{i j}\right\}_{i, j=1}^{2}$ is a symmetric matrix whose entries $a_{i j}$ are bounded, piecewise continuous real-valued functions defined on $\bar{\Omega}$, with

$$
\zeta^{T} a(x) \zeta>0 \quad \forall \zeta \in \mathbb{R}^{2}, \quad \text { a.e. } x \in \bar{\Omega} .
$$




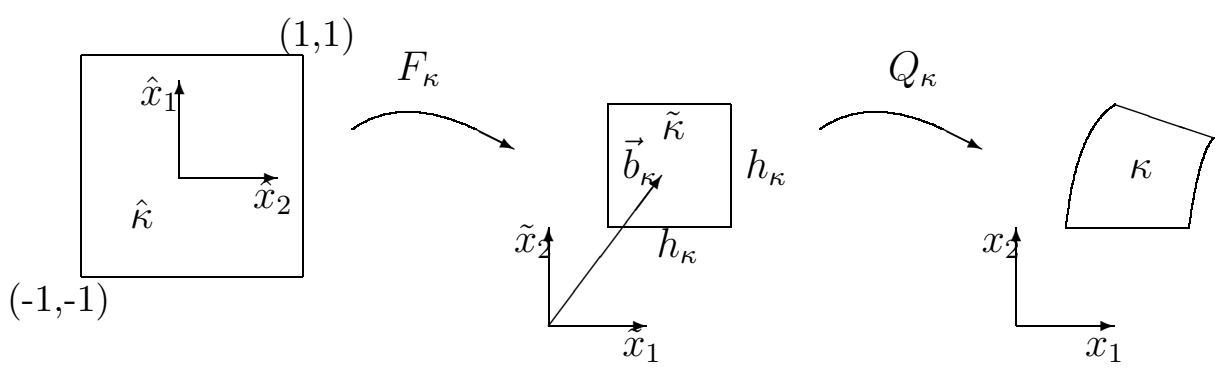

Figure 1: Construction of elements via composition of affine maps and diffeomorphisms.

We decompose $\Gamma_{\partial}$ into two parts $\Gamma_{\mathrm{D}}, \Gamma_{\mathrm{N}}$ on which we impose Dirichlet and Neumann boundary conditions respectively

$$
\begin{aligned}
u & =g_{\mathrm{D}} \text { on } \Gamma_{\mathrm{D}} \\
(a \nabla u) \cdot \mu & =g_{\mathrm{N}} \text { on } \Gamma_{\mathrm{N}} .
\end{aligned}
$$

\section{Function Spaces}

We shall denote by $H^{s}(\Omega)$ the standard Hilbertian Sobolev space of index $s \geq 0$ of real-valued functions defined on $\Omega$.

Let $\mathcal{T}$ be a subdivision of the polygonal domain $\Omega$ into disjoint open elements $\kappa$ constructed via mappings $Q_{\kappa} \circ F_{\kappa}$, where $F_{\kappa}: \hat{\kappa}:=(-1,1)^{2} \rightarrow \tilde{\kappa}$ is an affine mapping of the form

$$
F_{\kappa}(\vec{x}):=A_{\kappa} \vec{x}+\vec{b}_{\kappa}
$$

with $A_{\kappa}:=\left(h_{\kappa} / 2\right) \mathrm{id}_{2}, \mathrm{id}_{2}$ the $2 \times 2$ identity matrix, $\vec{b}_{\kappa}$ a two-component real-valued vector, and $Q_{\kappa}: \tilde{\kappa} \rightarrow \kappa$ a $C^{1}$-diffeomorphism (cf. Figure 1 ).

Heuristically, we can say that the affine mapping $F_{\kappa}$ defines the size of the element $\kappa$ and the diffeomorphism $Q_{\kappa}$ defines the "shape". For this reason, we shall be working with diffeomorphisms that are close to the identity in the following sense: the Jacobian $J_{Q_{\kappa}}$ of $Q_{\kappa}$ satisfies

$$
C_{1}^{-1} \leq \operatorname{det} J_{Q_{\kappa}} \leq C_{1}, \quad\left\|\left(J_{Q_{\kappa}}\right)_{i j}\right\|_{L^{\infty}(\kappa)} \leq C_{2}, i, j=1,2, \text { for all } \kappa \in \mathcal{T},
$$

for some positive constants $C_{1}, C_{2}$, independent of the meshsize $h_{\kappa}:=\operatorname{diam}(\kappa)$.

The above maps are assumed to be constructed so as to ensure that the union of the closures of the elements $\kappa \in \mathcal{T}$ forms a covering of the closure of $\Omega$, i.e., $\bar{\Omega}=\cup_{\kappa \in \mathcal{T}} \bar{\kappa}$.

In the approximation theory presented below, another kind of space will prove to be useful, in order to ensure the optimality of subsequent error bounds. More specifically, we introduce the notion of an augmented Sobolev space. 

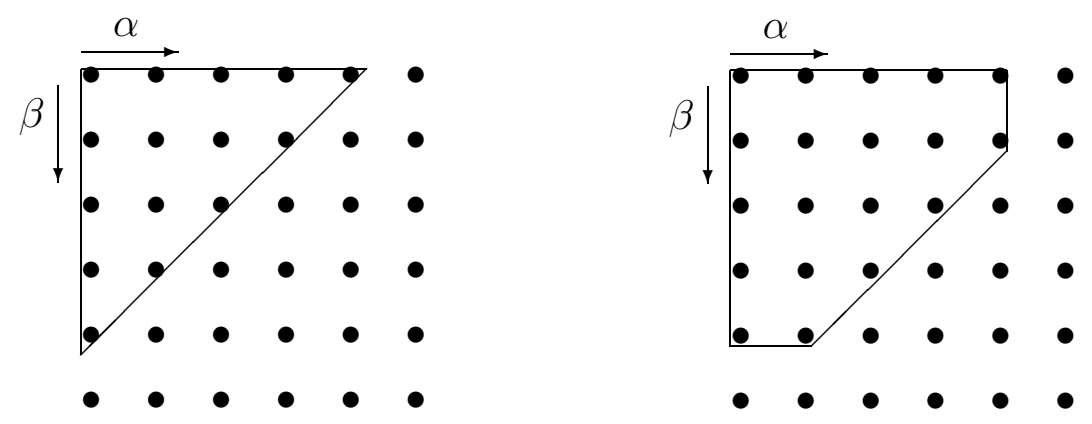

(Standard) Sobolev space $H^{s}, s=5$ Augmented Sobolev space $\tilde{H}^{s}, s=5$

Figure 2: Sobolev index diagrams for different kinds of Sobolev spaces.

Definition 1 Let $s$ be a nonnegative integer and $\kappa$ an open (curvilinear) quadrilateral domain constructed as the image of an axiparallel rectangle $\tilde{\kappa}$ through a $C^{s+1}$ diffeomorphism $Q_{\kappa}$, as indicated above. We define the augmented Sobolev space of order $s$ on $\kappa$ by

$$
\tilde{H}^{s}(\kappa):=\left\{\left(u \circ Q_{\kappa}\right) \in H^{s}(\tilde{\kappa}): \text { for }(\alpha, \beta) \in I_{A}, \partial_{1}^{\alpha} \partial_{2}^{\beta}\left(u \circ Q_{\kappa}\right) \in L^{2}(\tilde{\kappa})\right\},
$$

where

$$
I_{A}:=\{(\alpha, \beta): \alpha+\beta=s+1 \text {, excluding }(s+1,0) \text { and }(0, s+1)\},
$$

with associated norm $\|\cdot\|_{\tilde{H}^{s}(\kappa)}$ and seminorm $|\cdot|_{\tilde{H}^{s}(\kappa)}$ :

$$
\begin{aligned}
\|u\|_{\tilde{H}^{s}(\kappa)} & :=\left(\left\|u \circ Q_{\kappa}\right\|_{H^{s}(\tilde{\kappa})}^{2}+\sum_{(\alpha, \beta) \in I_{A}}\left\|\partial_{1}^{\alpha} \partial_{2}^{\beta}\left(u \circ Q_{\kappa}\right)\right\|_{\tilde{\kappa}}^{2}\right)^{\frac{1}{2}}, \\
|u|_{\tilde{H}^{s}(\kappa)} & :=\left(\left\|\partial_{1}^{s}\left(u \circ Q_{\kappa}\right)\right\|_{\tilde{\kappa}}^{2}+\left\|\partial_{2}^{s}\left(u \circ Q_{\kappa}\right)\right\|_{\tilde{\kappa}}^{2}+\sum_{(\alpha, \beta) \in I_{A}} h_{\kappa}\left\|\partial_{1}^{\alpha} \partial_{2}^{\beta}\left(u \circ Q_{\kappa}\right)\right\|_{\tilde{\kappa}}^{2}\right)^{\frac{1}{2}} .
\end{aligned}
$$

Clearly, the imbedding $\tilde{H}^{s}(\kappa) \hookrightarrow H^{s}(\kappa)$ holds. For a schematic representation of these spaces in terms of Sobolev indices, we refer to Figure 2. The above definition can be extended to include augmented Sobolev spaces with nonnegative real Sobolev indices, using a standard function space interpolation argument.

We discuss briefly this somewhat non-standard construction. The space $\tilde{H}^{s}(\kappa)$, equipped with the corresponding natural inner-product, is a Hilbert space. Note that $\tilde{H}^{s}$ depends on the diffeomorphism $Q_{\kappa}$, and therefore $\tilde{H}_{Q_{\kappa}}^{s}(\kappa)$ would have been a more accurate notation for the space. We have chosen to omit the subscript, as the choice of diffeomorphism $Q_{\kappa}$ will always be clear from the context. We note that in contrast with standard Sobolev space regularity (see, e.g., Theorem 4.1 in [11]), augmented Sobolev space regularity is not invariant under diffeomorphisms. This can be easily seen by 
choosing $Q_{\kappa}$ to be a rotation; then the chain rule implies that for $u \circ Q_{\kappa} \in \tilde{H}^{s}(\tilde{\kappa})$ we need in general $u \in H^{s+1}(\kappa)$. The maps $Q_{\kappa}$ are on the other hand needed to prove approximation estimates for the $H^{1}$-projection operator defined below. Indeed, as the standard procedure of deriving such estimates is by mapping to some reference domain $\tilde{\kappa}$, a way to overcome this lack of invariance is to demand the additional regularity on the mapped domain $\kappa$ instead. This idea is also applicable when shape-irregular (anisotropic) elements are considered; this case though will be treated elsewhere.

Since the $h p$-DGFEM is a non-conforming method, it is necessary to introduce the notion of a broken Sobolev space.

Definition 2 We define the broken Sobolev space of composite order $\boldsymbol{s}$ on an open set $\Omega$, subject to a subdivision $\mathcal{T}$ of $\Omega$, as

$$
H^{\boldsymbol{s}}(\Omega, \mathcal{T})=\left\{u \in L^{2}(\Omega):\left.u\right|_{\kappa} \in \mathcal{H}^{s_{\kappa}}(\kappa) \forall \kappa \in \mathcal{T}\right\}
$$

with $\mathcal{H}^{s_{\kappa}}(\kappa) \in\left\{H^{s_{\kappa}}(\kappa), \tilde{H}^{s_{\kappa}}(\kappa)\right\}, s_{\kappa}$ being the local Sobolev index on the element $\kappa$, and $s:=\left(s_{\kappa}: \kappa \in \mathcal{T}\right)$; when $s_{\kappa}=s$ for all $\kappa \in \mathcal{T}$, we shall write $H^{s}(\Omega, \mathcal{T})$.

\section{Weak Formulation}

Let us introduce some notation first. We denote by $\mathcal{T}$ a subdivision of $\Omega$ into elements $\kappa$. By $\mathcal{E}$ we denote the set of all open one-dimensional element faces associated with the subdivision $\mathcal{T}$, and we define $\Gamma:=\cup_{e \in \mathcal{E}} e$. We also assume that $\mathcal{E}$ is decomposed into two subsets, namely $\mathcal{E}_{\text {int }}$ and $\mathcal{E}_{\partial}$, which contain the set of all elements of $\mathcal{E}$ that are not subsets of $\partial \Omega$ and the set of all elements of $\mathcal{E}$ that are subsets of $\partial \Omega$, respectively. $\mathcal{E}_{\partial}$ is further decomposed into $\mathcal{E}_{\mathrm{D}}$ and $\mathcal{E}_{\mathrm{N}}$, and we define $\Gamma_{\mathrm{D}}:=\cup_{e \in \mathcal{E}_{\mathrm{D}}} e, \Gamma_{\mathrm{N}}:=\cup_{e \in \mathcal{E}_{\mathrm{N}}} e$ and $\Gamma_{\text {int }}:=\cup_{e \in \mathcal{E}_{\text {int }}} e$, all with the obvious meanings. Thus, introducing an element numbering, $1, \ldots,|\mathcal{T}|$, and given an interface $e \in \mathcal{E}_{\text {int }}$, there exist indices $i$ and $j$ such that $i>j$ and the elements $\kappa:=\kappa_{i}$ and $\kappa^{\prime}:=\kappa_{j}$ share the edge $e$. We define the jump of a function $u \in H^{1}(\Omega, \mathcal{T})$ across $e$ and the mean value of $u$ on $e$ by

$$
[u]_{e}:=\left.u\right|_{\partial \kappa \cap e}-\left.u\right|_{\partial \kappa^{\prime} \cap e} \text { and }\langle u\rangle_{e}:=\frac{1}{2}\left(\left.u\right|_{\partial \kappa \cap e}+\left.u\right|_{\partial \kappa^{\prime} \cap e}\right),
$$

respectively, where $\partial \kappa$ denotes the union of all open edges of the element $\kappa$. The subscript $e$ in these definitions will be suppressed when no confusion is likely to occur. With each edge we associate the unit normal vector $\nu$ pointing from element $\kappa_{i}$ to $\kappa_{j}$ when $i>j$; we choose $\nu$ to be the unit outward normal $\mu$ when the edge belongs to $\mathcal{E}_{\partial}$.

The "broken" weak formulation for the problem (2.1), (2.3), from which the interior penalty DGFEM will emerge, reads

$$
\text { Find } u \in A \text { such that } B(u, v)=l(v) \quad \forall v \in H^{2}(\Omega, \mathcal{T}) \text {, }
$$

where

$$
A:=\left\{u \in H^{2}(\Omega, \mathcal{T}): u,(a \nabla u) \cdot \nu \text { are continuous across } e \in \mathcal{E}_{\text {int }}\right\}
$$




$$
\begin{aligned}
B(u, v):= & \sum_{\kappa \in \mathcal{T}} \int_{\kappa}(a \nabla u \cdot \nabla v+c u v) \mathrm{d} x \\
& +\int_{\Gamma_{\mathrm{D}}}\{\theta u((a \nabla v) \cdot \mu)-((a \nabla u) \cdot \mu) v+\sigma u v\} \mathrm{d} s \\
& +\int_{\Gamma_{\mathrm{int}}}\{\theta[u]\langle(a \nabla v) \cdot \nu\rangle-\langle(a \nabla u) \cdot \nu\rangle[v]+\sigma[u][v]\} \mathrm{d} s,
\end{aligned}
$$

and

$$
l(v):=\sum_{\kappa \in \mathcal{T}} \int_{\kappa} f v \mathrm{~d} x+\int_{\Gamma_{\mathrm{D}}}\left\{\theta g_{\mathrm{D}}((a \nabla v) \cdot \mu)+\sigma g_{\mathrm{D}} v\right\} \mathrm{d} s+\int_{\Gamma_{\mathrm{N}}} g_{\mathrm{N}} v \mathrm{~d} s,
$$

with $\theta \in\{-1,1\}$, and the function $\sigma$ to be defined in the sequel. This weak formulation will be referred to as the broken weak formulation. Note that, if $\theta=-1$ the bilinear form $B(\cdot, \cdot)$ is symmetric; this is not the case, though, if $\theta=1$.

\section{Discontinuous Galerkin Finite Element Method}

Adopting the notation of Section 3, we introduce the following definition.

Definition 3 Let $\mathbf{p}:=\left(p_{\kappa}: \kappa \in \mathcal{T}\right)$ be the vector containing the polynomial degrees of the elements in a given subdivision $\mathcal{T}$. We define the finite element space with respect to $\Omega, \mathcal{T}, \mathbf{F}:=\left(F_{\kappa}: \kappa \in \mathcal{T}\right), \mathbf{Q}:=\left(Q_{\kappa}: \kappa \in \mathcal{T}\right)$ and $\mathbf{p}$ by

$$
S^{\mathbf{p}}(\Omega, \mathcal{T}, \mathbf{F}, \mathbf{Q}):=\left\{u \in L^{2}(\Omega):\left.u\right|_{\kappa} \circ Q_{\kappa} \circ F_{\kappa} \in \mathcal{Q}_{p_{\kappa}}(\hat{\kappa})\right\} .
$$

Here $\mathcal{Q}_{p_{\kappa}}(\hat{\kappa})$ is the space of tensor-product polynomials on $\hat{\kappa}$ of degree at most $p_{\kappa}$.

Using the weak formulation derived in Section 4 and the finite element spaces constructed in the previous section, the discontinuous Galerkin finite element method for the problem $(2.1),(2.3)$ reads:

Find $u_{\mathrm{DG}} \in S^{\mathbf{p}}(\Omega, \mathcal{T}, \mathbf{F}, \mathbf{Q})$ such that $B\left(u_{\mathrm{DG}}, v\right)=l(v) \quad \forall v \in S^{\mathbf{p}}(\Omega, \mathcal{T}, \mathbf{F}, \mathbf{Q})$,

with the function $\sigma$ contained in $B(\cdot, \cdot)$ and $l(\cdot)$ to be defined in the error analysis. If $\theta=-1$ we shall refer to the method as the symmetric version, and if $\theta=1$ we shall speak about (5.1) as the non-symmetric version of the $h p$-DGFEM. The terminology stems from the fact that the bilinear form $B(\cdot, \cdot)$ is symmetric if, and only if, $\theta=-1$.

We make some assumptions on the regularity of the solution and on the functions in the finite element space $S^{\mathbf{p}}(\Omega, \mathcal{T}, \mathbf{F}, \mathbf{Q})$. We assume that $p_{i}^{\kappa} \geq 1, i=1,2, \kappa \in \mathcal{T}$, in order to ensure the non-degeneracy of the linear system. In the error analysis we shall require the Galerkin orthogonality property: $B\left(u-u_{\mathrm{DG}}, v\right)=0, \forall v \in S^{\mathbf{p}}(\Omega, \mathcal{T}, \mathbf{F}, \mathbf{Q})$, where $u$ is the analytical solution of the problem and $u_{\mathrm{DG}}$ is the discontinuous Galerkin approximation to $u$ defined by the method (5.1). If these continuity assumptions are violated, as for example in an elliptic transmission problem, the DGFEM has to be modified accordingly. 


\section{$6 \quad H^{1}$-Projection Operator}

We begin with some prerequisites.

Lemma 1 Let $u \in H^{k+1}(\hat{\kappa}), k \geq 0$. Then the following trace inequality holds:

$$
\|u\|_{\partial \hat{\kappa}_{i}}^{2} \leq\|u\|_{\hat{\kappa}}^{2}+4\|u\|_{\hat{\kappa}}\left\|\partial_{j} u\right\|_{\hat{\kappa}}, \quad i, j \in\{1,2\}, i \neq j
$$

where $\partial \hat{\kappa}_{1}:=(-1,1) \times\{ \pm 1\}, \partial \hat{\kappa}_{2}:=\{ \pm 1\} \times(-1,1)$, and $\partial_{j} u:=\partial u / \partial x_{j}, j \in\{1,2\}$.

For a proof see, e.g., [3].

Definition 4 We define the $H^{1}$-projection operator $\pi_{p}: H^{1}(\hat{I}) \rightarrow \mathcal{P}_{p}(\hat{I}), p \geq 1, \mathcal{P}_{p}(\hat{I})$ being the space of polynomials of degree at most $p$ on $\hat{I}$, by setting, for $\hat{u} \in H^{1}(\hat{I})$,

$$
\left(\pi_{p} \hat{u}\right)(x):=\int_{-1}^{x} \tilde{\pi}_{p-1}\left(\hat{u}^{\prime}\right)(\eta) \mathrm{d} \eta+\hat{u}(-1), \quad x \in \hat{I}
$$

with $\tilde{\pi}_{p-1}$ being the $L^{2}$-projection operator onto $\mathcal{P}_{p-1}(\hat{I})$.

We introduce some notation. We denote by $\Phi_{1}(p, s)$ and $\Phi_{2}(p, s)$ the quantities

$$
\Phi_{1}(p, s):=\left(\frac{\Gamma(p-s+1)}{\Gamma(p+s+1)}\right)^{\frac{1}{2}} \text { and } \quad \Phi_{2}(p, s):=\frac{\Phi_{1}(p, s)}{\sqrt{p(p+1)}}
$$

respectively, with $p, s$ real numbers such that $0 \leq s \leq p$ and $\Gamma(\cdot)$ being the Gamma function (see, e.g., [7]); we also adopt the standard convention $\Gamma(1)=0$ ! $=1$. We remark on the asymptotic behaviour of $\Phi_{1}(p, s)$. Making use of Stirling's formula (see, e.g., [7]),

$$
\Gamma(n) \sim \sqrt{2 \pi} n^{n-\frac{1}{2}} e^{-n}, \quad n>0
$$

we can see that, for $p \geq 1$,

$$
\Phi_{1}(p, s)=\left(\frac{\Gamma(p-s+1)}{\Gamma(p+s+1)}\right)^{\frac{1}{2}} \leq C(s) p^{-s}
$$

with $0 \leq s \leq p$ and $C(s)$ denotes a constant depending on $s$ only.

We shall make use of the following result for the $L^{2}$-projection operator in one variable, taken from [5].

Lemma 2 For $\hat{I}:=(-1,1)$, let $\hat{u} \in H^{k+1}(\hat{I})$ for some integer $k \geq 0$ and let $\tilde{\pi}_{p} \hat{u} \in \mathcal{P}_{p}(\hat{I})$ be its $L^{2}$-projection with $p \geq 0$, where $\mathcal{P}_{p}(\hat{I})$ is the space of polynomials on $\hat{I}$ of degree at most $p$; then

$$
\left|\left(\hat{u}-\tilde{\pi}_{p} \hat{u}\right)( \pm 1)\right| \leq \frac{\Phi_{1}(p, t)}{\sqrt{2 p+1}}\left\|\hat{u}^{(t+1)}\right\|_{\hat{I}}
$$

for any integer $t, 0 \leq t \leq \min \{p, k\}, p \geq 0$ and $\Phi_{1}$ as defined above. 
We recall the following one-dimensional approximation result from [9] for the $H^{1}$ projection.

Theorem 3 Let $p \geq 1, k \geq 0$ and $\hat{u} \in H^{k+1}(\hat{I})$; then, for the $H^{1}$-projection $\pi_{p} \hat{u} \in \mathcal{P}_{p}(\hat{I})$ of $\hat{u}$ defined above, the following hold:

$$
\begin{gathered}
\pi_{p} \hat{u}( \pm 1)=\hat{u}( \pm 1), \\
\left\|\hat{u}^{\prime}-\left(\pi_{p} \hat{u}\right)^{\prime}\right\|_{\hat{I}} \leq \Phi_{1}(p, s)\left\|\hat{u}^{(s+1)}\right\|_{\hat{I}},
\end{gathered}
$$

for any $0 \leq s \leq \min \{p, k\}$, and

$$
\left\|\hat{u}-\pi_{p} \hat{u}\right\|_{\hat{I}} \leq \Phi_{2}(p, t)\left\|\hat{u}^{(t+1)}\right\|_{\hat{I}}
$$

for any $0 \leq t \leq \min \{p, k\}$.

Proof See the proofs of Theorem 3.14 and Corollary 3.15 in [9].

Corollary 3A Let $\hat{u} \in H^{1}(\hat{I})$; then the projection operator $\pi_{p}$ obeys the following bounds:

$$
\begin{gathered}
\left\|\left(\pi_{p} \hat{u}\right)^{\prime}\right\|_{\hat{I}} \leq\left\|\hat{u}^{\prime}\right\|_{\hat{I}}, \\
\left\|\pi_{p} \hat{u}\right\|_{\hat{I}} \leq\|\hat{u}\|_{\hat{I}}+\Phi_{2}(p, 0)\left\|\hat{u}^{\prime}\right\|_{\hat{I}},
\end{gathered}
$$

for all $p \geq 1$.

We shall now extend the results described above to the two-dimensional setting.

Definition 5 Let $\hat{\kappa} \equiv(-1,1)^{2}$ and $\hat{u} \in \tilde{H}^{1}(\hat{\kappa})$. We define the tensor-product projection operator $\pi_{p}$, with polynomial degree $p$, by

$$
\pi_{\vec{p}}=\pi_{p}^{1} \pi_{p}^{2}:=\left(\pi_{p}^{1} \otimes I\right) \circ\left(I \otimes \pi_{p}^{2}\right)
$$

with $\otimes$ denoting the standard tensor product, where $\pi_{p}^{1}, \pi_{p}^{2}$ denote the one-dimensional $H^{1}$-projection operators from Definition 4, and the superscripts 1,2 indicate the directions in which these one-dimensional projections are applied.

In fact, one can easily prove that

$$
\partial_{1} \pi_{p}^{2} \hat{u}=\pi_{p}^{2} \partial_{1} \hat{u} \text { and } \partial_{2} \pi_{p}^{1} \hat{u}=\pi_{p}^{1} \partial_{2} \hat{u}
$$

with $\partial_{i}, i \in\{1,2\}$ denoting the partial derivative in the $i$-th direction, and that

$$
\pi_{p}=\pi_{p}^{1} \pi_{p}^{2}=\pi_{p}^{2} \pi_{p}^{1}
$$

these properties will be useful in the derivation of our approximation results. 


\subsection{Approximation on the Reference Element}

We begin by discussing bounds on the approximation error in the $L^{2}$ - and $H^{1}$-norms.

Lemma 4 Let $\hat{u} \in \tilde{H}^{k+1}(\hat{\kappa}), k \geq 0$. Then the following hold:

$$
\begin{gathered}
\pi_{p} \hat{u}=\hat{u} \text { at the vertices of } \hat{\kappa} \\
\left.\pi_{p} \hat{u}\right|_{\partial \hat{\kappa}_{i}}=\pi_{p}^{i}\left(\left.\hat{u}\right|_{\partial \hat{\kappa}_{i}}\right)
\end{gathered}
$$

with $i \in\{1,2\}$ and $\partial \hat{\kappa}_{i}$ as above. Moreover,

$$
\left\|\hat{u}-\pi_{p} \hat{u}\right\|_{\hat{\kappa}} \leq \sum_{i=1}^{2} \Phi_{2}(p, s)\left\|\partial_{i}^{s+1} \hat{u}\right\|_{\hat{\kappa}}+\min _{i \in\{1,2\}}\left\{\Phi_{2}(p, 0) \Phi_{2}(p, t)\left\|\partial_{j}^{t+1} \partial_{i} \hat{u}\right\|_{\hat{\kappa}}\right\},
$$

and

$$
\left\|\partial_{i}\left(\hat{u}-\pi_{p} \hat{u}\right)\right\|_{\hat{\kappa}} \leq \Phi_{1}(p, s)\left\|\partial_{i}^{s+1} \hat{u}\right\|_{\hat{\kappa}}+\Phi_{2}(p, t)\left\|\partial_{j}^{t+1} \partial_{i} \hat{u}\right\|_{\hat{\kappa}},
$$

where $i, j \in\{1,2\}, i \neq j, 0 \leq s, t \leq \min \{p, k\}$, and $p \geq 1$. Furthermore, if $\hat{u} \in H^{k+1}(\hat{\kappa})$, with $k \geq 1$, then the bounds (8) and (6.12) hold for $0 \leq s \leq \min \{p, k\}$ and $0 \leq t \leq$ $\min \{p, k-1\}$.

Proof See Theorem 3.3 in [4] for the proof in the case of standard Sobolev spaces; the corresponding proof for augmented spaces follows analogously.

Lemma 5 Let $\hat{u} \in \tilde{H}^{k+1}(\hat{\kappa}), k \geq 0$. Then the following error estimate holds:

$$
\left\|\hat{u}-\pi_{p} \hat{u}\right\|_{\partial \hat{\kappa}_{i}} \leq\left(2 c_{1}^{-2}+1\right)^{\frac{1}{2}} \Phi_{2}(p, s)\left\|\partial_{i}^{s+1} \hat{u}\right\|_{\hat{\kappa}}+\sqrt{2} c_{1} \Phi_{2}(p, t)\left\|\partial_{i}^{t+1} \partial_{j} \hat{u}\right\|_{\hat{\kappa}},
$$

for $i, j \in\{1,2\}, i \neq j$, where $c_{1}$ is an arbitrary positive real number, $0 \leq s, t \leq \min \{p, k\}$, and $p \geq 1$. Furthermore, if $\hat{u} \in H^{k+1}(\hat{\kappa})$, with $k \geq 1$, then (6.13) holds for $0 \leq s \leq$ $\min \{p, k\}, 0 \leq t \leq \min \{p, k-1\}$.

Proof We prove (6.13) for $i=1$; for $i=2$ the proof is analogous. Using the trace inequality (6.1), we have

$$
\left\|\hat{u}-\pi_{p}^{1} \hat{u}\right\|_{\partial \hat{\kappa}_{1}}^{2} \leq\left\|\hat{u}-\pi_{p}^{1} \hat{u}\right\|_{\hat{\kappa}}^{2}+4\left\|\hat{u}-\pi_{p}^{1} \hat{u}\right\|_{\hat{\kappa}}\left\|\partial_{2} \hat{u}-\partial_{2}\left(\pi_{p}^{1} \hat{u}\right)\right\|_{\hat{\kappa}} .
$$

Recalling that $\partial_{2} \pi_{p}^{1}=\pi_{p}^{1} \partial_{2}$, using the inequality $2 \beta \gamma \leq \beta^{2} / c_{1}^{2}+c_{1}^{2} \gamma^{2}$, with $c_{1}>0$, for the second term on the right-hand side of (6.14), as well as the inequality $\sqrt{\alpha+\beta} \leq \sqrt{\alpha}+\sqrt{\beta}$, we get

$$
\left\|\hat{u}-\pi_{p}^{1} \hat{u}\right\|_{\partial \hat{\kappa}_{1}} \leq\left(\frac{2}{c_{1}^{2}}+1\right)^{\frac{1}{2}}\left\|\hat{u}-\pi_{p}^{1} \hat{u}\right\|_{\hat{\kappa}}+\sqrt{2} c_{1}\left\|\left(\partial_{2} \hat{u}\right)-\pi_{p}^{1}\left(\partial_{2} \hat{u}\right)\right\|_{\hat{\kappa}} .
$$

Now, applying (6.8) to each term on the right-hand side of (6.15), we obtain the result.

Next, we derive bounds for the $H^{1}$-approximation error on the boundary. 
Lemma 6 Let $\hat{u} \in \tilde{H}^{k+1}(\hat{\kappa}), k \geq 1$. Then the following error estimates hold:

$$
\begin{aligned}
\left\|\partial_{i}\left(\hat{u}-\pi_{p} \hat{u}\right)\right\|_{\partial \hat{\kappa}_{i}} & \leq\left(2 c_{2}^{-2}+1\right)^{\frac{1}{2}} \Phi_{1}(p, s)\left\|\partial_{i}^{s+1} \hat{u}\right\|_{\hat{\kappa}}+\sqrt{2} c_{2} \Phi_{1}(p, t)\left\|\partial_{i}^{t+1} \partial_{j} \hat{u}\right\|_{\hat{\kappa}} \\
\left\|\partial_{j}\left(\hat{u}-\pi_{p} \hat{u}\right)\right\|_{\partial \hat{\kappa}_{i}} & \leq\left(2 c_{3}^{-2}+1\right)^{\frac{1}{2}} \Phi_{2}(p, t)\left\|\partial_{i}^{t+1} \partial_{j} \hat{u}\right\|_{\hat{\kappa}}+\sqrt{2} c_{3} \Phi_{2}(p, q)\left\|\partial_{i}^{q+1} \partial_{j}^{2} \hat{u}\right\|_{\hat{\kappa}} \\
& +2 \frac{\Phi_{1}(p-1, r)}{\sqrt{2 p-1}}\left\|\partial_{j}^{r+2} \hat{u}\right\|_{\hat{\kappa}}+2 \Phi_{2}(p, 0) \frac{\Phi_{1}(p-1, z)}{\sqrt{2 p-1}}\left\|\partial_{i} \partial_{j}^{z+2} \hat{u}\right\|_{\hat{\kappa}},
\end{aligned}
$$

for $i, j \in\{1,2\}, i \neq j$, where $c_{2}, c_{3}$ are arbitrary positive real numbers, $0 \leq s, t \leq$ $\min \{p, k\}, 0 \leq q \leq \min \{p, k-1\}$ and $0 \leq r, z \leq \min \{p-1, k-1\}$. Moreover, if $\hat{u} \in H^{k+1}(\hat{\kappa})$, with $k \geq 2$, then (6.16) and (6.17) hold for $0 \leq s \leq \min \{p, k\}, 0 \leq$ $t \leq \min \{p, k-1\}, 0 \leq q \leq \min \{p, k-2\}, 0 \leq r \leq \min \{p-1, k-1\}$ and $0 \leq z \leq$ $\min \{p-1, k-2\}$.

Proof We prove the inequality (6.16) for $i=1$; for $i=2$ the proof is analogous. We have

$$
\left\|\partial_{1}\left(\hat{u}-\pi_{p} \hat{u}\right)\right\|_{\partial \hat{\kappa}_{1}}=\left\|\partial_{1} \hat{u}-\partial_{1} \pi_{p_{1}}^{1} \hat{u}\right\|_{\partial \hat{\kappa}_{1}},
$$

since $\pi_{p}^{1} \hat{u}=\pi_{p} \hat{u}$ on $\partial \hat{\kappa}_{1}$. Using the trace inequality (6.1), we have

$$
\begin{aligned}
\left\|\partial_{1} \hat{u}-\partial_{1} \pi_{p_{1}}^{1} \hat{u}\right\|_{\partial \hat{\kappa}_{1} \leq}^{2} \leq & \left\|\partial_{1} \hat{u}-\partial_{1} \pi_{p_{1}}^{1} \hat{u}\right\|_{\hat{\kappa}}^{2} \\
& +4\left\|\partial_{1} \hat{u}-\partial_{1} \pi_{p_{1}}^{1} \hat{u}\right\|_{\hat{\kappa}}\left\|\partial_{2} \partial_{1} \hat{u}-\partial_{2} \partial_{1}\left(\pi_{p}^{1} \hat{u}\right)\right\|_{\hat{\kappa}} .
\end{aligned}
$$

Recalling that $\partial_{2}$ commutes with both $\partial_{1}$ and $\pi_{p_{1}}^{1}$, using the inequality $2 \beta \gamma \leq \beta^{2} / c_{2}^{2}+c_{2}^{2} \gamma^{2}$ for the second term on the right-hand side of (6.19), as well as the inequality $\sqrt{\alpha+\beta} \leq \sqrt{\alpha}+\sqrt{\beta}$, we get

$$
\left\|\partial_{1}\left(\hat{u}-\pi_{p}^{1} \hat{u}\right)\right\|_{\partial \hat{\kappa}_{1}} \leq\left(\frac{2}{c_{2}^{2}}+1\right)^{\frac{1}{2}}\left\|\partial_{1}\left(\hat{u}-\pi_{p}^{1} \hat{u}\right)\right\|_{\hat{\kappa}}+\sqrt{2} c_{2}\left\|\partial_{1}\left(\left(\partial_{2} \hat{u}\right)-\pi_{p}^{1}\left(\partial_{2} \hat{u}\right)\right)\right\|_{\hat{\kappa}} .
$$

Applying (6.7) to the terms on the right-hand side of (6.20), we obtain the result.

Now, we prove the bound on the derivative in the second variable of the approximation error on $\partial \hat{\kappa}_{1}$ stated in (6.17) (case $i=1$ ). We have

$$
\left\|\partial_{2}\left(\hat{u}-\pi_{p} \hat{u}\right)\right\|_{\partial \hat{\kappa}_{1}} \leq\left\|\partial_{2} \hat{u}-\pi_{p}^{1} \partial_{2} \hat{u}\right\|_{\partial \hat{\kappa}_{1}}+\left\|\pi_{p}^{1} \partial_{2} \hat{u}-\partial_{2} \pi_{p}^{2}\left(\pi_{p}^{1} \hat{u}\right)\right\|_{\partial \hat{\kappa}_{1}}
$$

Applying a similar argument as above, the first term on the right-hand side can be bounded as follows:

$$
\left\|\partial_{2}\left(\hat{u}-\pi_{p}^{1} \hat{u}\right)\right\|_{\partial \hat{\kappa}_{1}} \leq\left(\frac{2}{c_{3}^{2}}+1\right)^{\frac{1}{2}}\left\|\left(\partial_{2} \hat{u}\right)-\pi_{p}^{1}\left(\partial_{2} \hat{u}\right)\right\|_{\hat{\kappa}}+\sqrt{2} c_{3}\left\|\left(\partial_{2}^{2} \hat{u}\right)-\pi_{p}^{1}\left(\partial_{2}^{2} \hat{u}\right)\right\|_{\hat{\kappa}} .
$$

In order to proceed, we observe that

$$
\pi_{p}^{1} \partial_{2} \hat{u}-\partial_{2} \pi_{p}^{2}\left(\pi_{p}^{1} \hat{u}\right)=\pi_{p}^{1}\left(\partial_{2} \hat{u}-\partial_{2} \pi_{p}^{2} \hat{u}\right)=\pi_{p}^{1}\left(\partial_{2} \hat{u}-\tilde{\pi}_{p-1}^{2} \partial_{2} \hat{u}\right)
$$


where $\tilde{\pi}_{p}^{2}$ denotes the $L^{2}$-projection operator in the second variable. Thus, using (6.10) and (6.5), as well as the (trivial) fact $\partial_{1} \tilde{\pi}_{p-1}^{2} \hat{u}=\tilde{\pi}_{p-1}^{2} \partial_{1} \hat{u}$, we have

$$
\begin{aligned}
\left\|\pi_{p}^{1} \partial_{2} \hat{u}-\partial_{2} \pi_{p}^{2}\left(\pi_{p}^{1} \hat{u}\right)\right\|_{\partial \hat{\kappa}_{1}}=\left\|\pi_{p}^{1}\left(\partial_{2} \hat{u}-\tilde{\pi}_{p-1}^{2} \partial_{2} \hat{u}\right)\right\|_{\partial \hat{\kappa}_{1}} \\
\leq\left\|\partial_{2} \hat{u}-\tilde{\pi}_{p-1}^{2} \partial_{2} \hat{u}\right\|_{\partial \hat{\kappa}_{1}}+\Phi_{2}(p, 0)\left\|\partial_{1}\left(\partial_{2} \hat{u}-\tilde{\pi}_{p-1}^{2} \partial_{2} \hat{u}\right)\right\|_{\partial \hat{\kappa}_{1}} \\
=\left(\int_{-1}^{1}\left|\partial_{2} \hat{u}-\tilde{\pi}_{p-1}^{2} \partial_{2} \hat{u}\right|^{2}\left(x_{1}, \pm 1\right) \mathrm{d} x_{1}\right)^{\frac{1}{2}} \\
+\Phi_{2}(p, 0)\left(\int_{-1}^{1}\left|\partial_{1} \partial_{2} \hat{u}-\tilde{\pi}_{p-1}^{2} \partial_{1} \partial_{2} \hat{u}\right|^{2}\left(x_{1}, \pm 1\right) \mathrm{d} x_{1}\right)^{\frac{1}{2}} \\
\leq 2 \frac{\Phi_{1}(p-1, r)}{\sqrt{2 p-1}}\left(\int_{-1}^{1} \int_{-1}^{1}\left(\partial_{2}^{r+2} \hat{u}\right)^{2}\left(x_{1}, x_{2}\right) \mathrm{d} x_{2} \mathrm{~d} x_{1}\right)^{\frac{1}{2}} \\
+2 \Phi_{2}(p, 0) \frac{\Phi_{1}(p-1, z)}{\sqrt{2 p-1}}\left(\int_{-1}^{1} \int_{-1}^{1}\left(\partial_{1} \partial_{2}^{z+2} \hat{u}\right)^{2}\left(x_{1}, x_{2}\right) \mathrm{d} x_{2} \mathrm{~d} x_{1}\right)^{\frac{1}{2}} \\
=2 \frac{\Phi_{1}(p-1, r)}{\sqrt{2 p-1}}\left\|\partial_{2}^{r+2} \hat{u}\right\|_{\hat{\kappa}}+2 \Phi_{2}(p, 0) \frac{\Phi_{1}(p-1, z)}{\sqrt{2 p-1}}\left\|\partial_{1} \partial_{2}^{z+2} \hat{u}\right\|_{\hat{\kappa}},
\end{aligned}
$$

with $0 \leq r, z \leq \min \{p-1, k-1\}$. The proof for $i=2$ is analogous.

\subsection{Approximation on general elements}

Having derived bounds on various norms of the projection error on the reference element $\hat{\kappa}$, we now present error estimates on axiparallel rectangular elements and on diffeomorphic images of those.

Definition 6 We define the tensor-product $H^{1}$-projection operator $\pi_{p}^{\tilde{h}}$ on $\tilde{\kappa}$, by

$$
\pi_{p}^{\tilde{h}} \tilde{u}:=\left(\pi_{p}\left(\tilde{u} \circ F_{\kappa}\right)\right) \circ F_{\kappa}^{-1}, \quad \tilde{u} \in H^{1}(\tilde{\kappa}),
$$

where, as before, $\pi_{p}$ denotes the tensor-product $H^{1}$-projector on the reference element $\hat{\kappa}$. Moreover, we define the tensor-product $H^{1}$-projection operator $\pi_{p}^{h}$ on $\kappa$, by

$$
\pi_{p}^{h} u:=\left(\pi_{p}^{\tilde{h}}\left(u \circ Q_{\kappa}\right)\right) \circ Q_{\kappa}^{-1}, \quad u \in H^{1}(\kappa) .
$$

Theorem 7 Let $\kappa$ be a shape-regular element and $h_{\kappa}:=\operatorname{diam}(\tilde{\kappa})$. Let also $u \in H^{1}(\kappa)$ be such that $\tilde{u}:=u \circ Q_{\kappa} \in \tilde{H}^{k+1}(\tilde{\kappa})$ with $k \geq 0$, and suppose that $p_{\kappa} \geq 1$. Then the following error estimates hold:

$$
\left\|u-\pi_{p_{\kappa}}^{h} u\right\|_{\kappa} \leq C\left(Q_{\kappa}\right) \Phi_{2}\left(p_{\kappa}, s_{\kappa}\right)\left(\frac{h_{\kappa}}{2}\right)^{s_{\kappa}+1}|u|_{\tilde{H}^{s_{\kappa}+1}(\kappa)},
$$

and

$$
\left\|\nabla\left(u-\pi_{p_{\kappa}}^{h} u\right)\right\|_{\kappa} \leq C\left(Q_{\kappa}\right) \Phi_{1}\left(p_{\kappa}, s_{\kappa}\right)\left(\frac{h_{\kappa}}{2}\right)^{s_{\kappa}}|u|_{\tilde{H}^{s_{\kappa}+1}(\kappa)} .
$$


If $u \in H^{k+1}(\kappa)$, for $k \geq 1$, then the following error estimates hold:

$$
\left\|u-\pi_{p_{\kappa}}^{h} u\right\|_{\kappa} \leq C\left(Q_{\kappa}\right) \Phi_{2}\left(p_{\kappa}, s_{\kappa}\right)\left(\frac{h_{\kappa}}{2}\right)^{s_{\kappa}+1}|u|_{H^{s_{\kappa}+1}(\kappa)},
$$

and

$$
\left\|\nabla\left(u-\pi_{p_{\kappa}}^{h} u\right)\right\|_{\kappa} \leq C\left(Q_{\kappa}\right) \Phi_{1}\left(p_{\kappa}, s_{\kappa}\right)\left(\frac{h_{\kappa}}{2}\right)^{s_{\kappa}}|u|_{H^{s_{\kappa}+1}(\kappa)} .
$$

Proof See Theorem 3.4 in [4] for the proof in the case of standard Sobolev spaces; the corresponding proof for augmented spaces follows analogously.

Theorem 8 Let $\kappa$ be a shape-regular element and $h_{\kappa}:=\operatorname{diam}(\tilde{\kappa})$, its diameter. Let also $u \in H^{1}(\Omega)$ be such that $\tilde{u}:=u \circ Q_{\kappa} \in \tilde{H}^{k+1}(\tilde{\kappa})$ with $k \geq 0$, and suppose that $p_{\kappa} \geq 1$. Then the following error estimate holds:

$$
\left\|u-\pi_{p_{\kappa}}^{h} u\right\|_{\partial \kappa} \leq C\left(Q_{\kappa}, \tilde{c}\right) \Phi_{2}\left(p_{\kappa}, s_{\kappa}\right)\left(\frac{h_{\kappa}}{2}\right)^{s_{\kappa}+\frac{1}{2}}|u|_{\tilde{H}^{s_{\kappa}+1}(\kappa)},
$$

with $0 \leq s_{\kappa} \leq \min \left\{p_{\kappa}, k\right\}, p_{\kappa} \geq 1$ and $\tilde{c}$ being the shape-regularity constant, defined as $\tilde{c}^{-2} \leq h_{i} / h_{j} \leq \tilde{c}^{2}$ for $i=1,2$.

If $u \in H^{k+1}(\kappa)$, with $k \geq 1$, then the following estimate holds:

$$
\left\|u-\pi_{p_{\kappa}}^{h} u\right\|_{\partial \kappa} \leq C\left(Q_{\kappa}, \tilde{c}\right) p_{\kappa}^{-\frac{1}{2}} \Phi_{1}\left(p_{\kappa}, s_{\kappa}\right)\left(\frac{h_{\kappa}}{2}\right)^{s_{\kappa}+\frac{1}{2}}|u|_{H^{s_{\kappa}+1}(\kappa)},
$$

with $1 \leq s_{\kappa} \leq \min \left\{p_{\kappa}, k\right\}, p_{\kappa} \geq 1$.

Proof After scaling to $\hat{\kappa}$, we choose $c_{1}=1$ in (5) for (6.26), and $c_{1}=p_{\kappa}^{-1}$ in (5) for (6.27). Finally, scaling back to $\tilde{\kappa}$ gives the results.

Remark 9 Making use of Stirling's formula, the bound (6.26) can be rewritten as

$$
\left\|u-\pi_{p_{\kappa}}^{h} u\right\|_{\partial \kappa} \leq C \frac{h_{\kappa}^{s_{\kappa}+1 / 2}}{p_{\kappa}^{s_{\kappa}+1}}|u|_{\tilde{H}^{s_{\kappa}+1}(\kappa)},
$$

with $0 \leq s_{\kappa} \leq \min \left\{p_{\kappa}, k\right\}$ and $C=C\left(Q_{\kappa}, \tilde{c}, s_{\kappa}\right)$. Hence, working in the augmented Sobolev space framework, we have enhanced convergence in the polynomial degree $p$ by half an order of $p$ beyond the optimal order of $p^{-\left(s_{\kappa}+1 / 2\right)}$. This property will be crucial for deriving our hp-optimal error bounds.

Finally, based on the estimates of Lemma 6 , we obtain the following results for the $H^{1}$-approximation on the element boundary. 
Theorem 10 Let $u \in \tilde{H}^{k+1}(\kappa)$, with $k \geq 1$; then the following estimate holds:

$$
\left\|\nabla\left(u-\pi_{p_{\kappa}}^{h} u\right)\right\|_{\partial \kappa} \leq C\left(Q_{\kappa}, \tilde{c}\right) p_{\kappa}^{\frac{1}{2}} \Phi_{1}\left(p_{\kappa}, s_{\kappa}\right)\left(\frac{h_{\kappa}}{2}\right)^{s_{\kappa}-\frac{1}{2}}|u|_{\tilde{H}^{s_{\kappa}+1}(\kappa)},
$$

with $1 \leq s_{\kappa} \leq \min \left\{p_{\kappa}, k\right\}, p_{\kappa} \geq 1$.

Let $u \in H^{k+1}(\kappa)$, with $k \geq 2$; then the following error estimate holds:

$$
\left\|\nabla\left(u-\pi_{p_{\kappa}}^{h} u\right)\right\|_{\partial \kappa} \leq C\left(Q_{\kappa}, \tilde{c}\right) p_{\kappa}^{\frac{1}{2}} \Phi_{1}\left(p_{\kappa}, s_{\kappa}\right)\left(\frac{h_{\kappa}}{2}\right)^{s_{\kappa}-\frac{1}{2}}|u|_{H^{s_{\kappa}+1}(\kappa)},
$$

with $2 \leq s_{\kappa} \leq \min \left\{p_{\kappa}, k\right\}, p_{\kappa} \geq 2$.

Proof First, we scale back to $\tilde{\kappa}$ using $Q_{\kappa}$; then using the chain rule and the triangle inequality of the norm, we have

$$
\begin{aligned}
\left\|\nabla\left(u-\pi_{p_{\kappa}}^{h} u\right)\right\|_{\partial \kappa} & \leq C\left(Q_{\kappa}, \tilde{c}\right) \sum_{i, j=1,2}\left\|\partial_{i}\left(\tilde{u}-\pi_{p_{\kappa}}^{\tilde{h}} \tilde{u}\right)\right\|_{\partial \tilde{\kappa}_{j}} \\
& \leq C\left(Q_{\kappa}, \tilde{c}\right)\left(\frac{2}{h_{\kappa}}\right)^{\frac{1}{2}} \sum_{i, j=1,2}\left\|\partial_{i}\left(\hat{u}-\pi_{p_{\kappa}} \hat{u}\right)\right\|_{\partial \hat{\kappa}_{j}} .
\end{aligned}
$$

If $\hat{u} \in \tilde{H}^{k+1}(\hat{\kappa})$, we first set $c_{3}=\left(2 p_{\kappa}\right)^{-1}, t_{i}=s_{i}$ and $q_{i}, r_{i}, z_{i}=s_{\kappa}-1$, for $i=1,2$, in (6.17). Next, using the bounds

$$
\Phi_{2}\left(p_{\kappa}, s_{\kappa}-1\right) \leq \Phi_{1}\left(p_{\kappa}, s_{\kappa}\right)
$$

and

$$
\frac{\Phi_{1}\left(p_{\kappa}-1, s_{\kappa}-1\right)}{\sqrt{2 p_{\kappa}-1}} \leq\left(2 p_{\kappa}\right)^{\frac{1}{2}} \Phi_{1}\left(p_{\kappa}, s_{\kappa}\right)
$$

and scaling back to $\tilde{\kappa}$, we deduce the result (6.29).

If $\hat{u} \in H^{k+1}(\hat{\kappa})$, we set $c_{2}=\left(2 p_{i}-1\right)^{-\frac{1}{2}}$ and $t_{i}=s_{\kappa}-1$, for $i=1,2$, and we use the relation

$$
\frac{\Phi_{1}\left(p_{\kappa}, s_{\kappa}-1\right)}{\sqrt{2 p_{\kappa}-1}} \leq\left(2 p_{\kappa}\right)^{\frac{1}{2}} \Phi_{1}\left(p_{\kappa}, s_{\kappa}\right)
$$

for $1 \leq s_{\kappa} \leq p_{\kappa}$. Also, we set $c_{3}=\left(p_{\kappa}-1\right)^{\frac{1}{2}}, t_{i}, z_{i}=s_{\kappa}-1$ and $q_{i}, r_{i}=s_{\kappa}-2$, for $i=1,2$. Then, applying (6.31) and the relations

$$
\begin{gathered}
\Phi_{2}\left(p_{\kappa}, s_{\kappa}-2\right) \leq\left(p_{\kappa}-1\right) \Phi_{1}\left(p_{\kappa}, s_{\kappa}\right), \\
\frac{\Phi_{1}\left(p_{\kappa}-1, s_{\kappa}-1\right)}{\sqrt{2 p_{\kappa}-1}} \leq\left(2 p_{\kappa}\right)^{\frac{1}{2}} \Phi_{1}\left(p_{\kappa}, s_{\kappa}\right)
\end{gathered}
$$

and

$$
\frac{\Phi_{1}\left(p_{\kappa}-1, s_{\kappa}-2\right)}{\sqrt{2 p_{\kappa}-1}} \leq \sqrt{2\left(p_{\kappa}-1\right)} \Phi_{1}\left(p_{\kappa}, s_{\kappa}-1\right),
$$

for $1 \leq s_{\kappa} \leq p_{\kappa}$, and scaling back to $\tilde{\kappa}$, we obtain the result (6.30). 


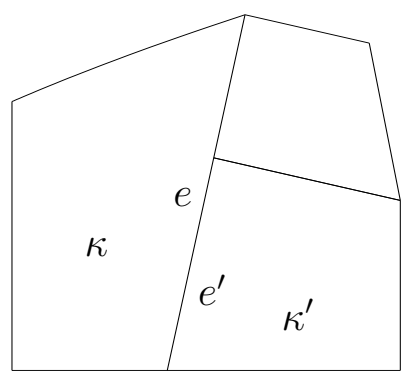

Figure 3: Hanging nodes

\section{Error analysis}

We shall make use of the above developments to derive improved error bounds for the $h p$-DGFEM.

Definition 7 Let $a, c$ and $B$ be as in (2.1) and (4.2) respectively. We define the functional $\mid\|\cdot \cdot\|_{\mathrm{DG}}$ on $H^{1}(\Omega, \mathcal{T})$, associated with the DGFEM, as follows:

$$
\|w\|_{\mathrm{DG}}:=\left(\sum_{\kappa \in \mathcal{T}}\left(\|\sqrt{a} \nabla w\|_{\kappa}^{2}+\|\sqrt{c} w\|_{\kappa}^{2}\right)+\int_{\Gamma_{\mathrm{D}}} \sigma w^{2} \mathrm{~d} s+\int_{\Gamma_{\mathrm{int}}} \sigma[w]^{2} \mathrm{~d} s\right)^{\frac{1}{2}},
$$

where $\sigma$ is a positive function defined on $\Gamma$.

It is easy to see that $\|\cdot \mid\|_{\mathrm{DG}}$ is a norm in $H^{1}(\Omega, \mathcal{T})$; we shall refer to it as the energy norm.

We shall assume elemental polynomial degrees $p_{\kappa}$ whose distribution obeys a bounded local variation condition, that is, there exists a constant $\rho \geq 1$ such that, for any pair of elements $\kappa$ and $\kappa^{\prime}$ which share an edge, we have

$$
\rho^{-1} \leq \frac{p_{\kappa}}{p_{\kappa^{\prime}}} \leq \rho
$$

Let meas $_{1}(\cdot)$ denote the one-dimensional Lebesgue measure. If

$$
\tau^{-1} \leq \operatorname{meas}_{1}(e) / \operatorname{meas}_{1}\left(e^{\prime}\right) \leq \tau,
$$

uniformly for all $e \in \mathcal{E}_{\text {int }}$ and all $e^{\prime} \in \mathcal{E}_{\text {int }}$ with $\operatorname{meas}_{1}\left(e \cap e^{\prime}\right)>0$ (cf. Figure 3), then we shall refer to such a subdivision $\mathcal{T}$ as regular, and to $\tau$ as the mesh regularity constant.

For simplicity of presentation, we shall assume that the entries of the diffusion tensor $a$ are such that $\sqrt{a} \in S^{\mathbf{q}}(\Omega, \mathcal{T}, \mathbf{F}, \mathbf{Q})$ where the composite polynomial degree vector $\mathbf{q}$ has nonnegative entries. Also, we define $\bar{a}_{\omega}:=\left\|\left(|\sqrt{a}|_{2}\right)^{2}\right\|_{L^{\infty}(\omega)}$ and $\gamma_{\omega}:=\|c\|_{L^{\infty}(\omega)}$, for any $\omega \subset \Omega$; here $|\cdot|_{2}$ denotes the matrix 2-norm. 
Theorem 11 Let $\Omega \subset \mathbb{R}^{2}$ be a (curvilinear) polygonal domain, $\mathcal{T}$ a regular subdivision of $\Omega$ into shape-regular elements constructed as in Section 5, possibly containing regular hanging nodes. We assign to every edge $e \in \mathcal{E}$ the positive real number

$$
\sigma_{e}:=C_{\sigma} \frac{\left\langle\bar{a}_{e} p^{2}\right\rangle_{e}}{h_{e}} \quad \text { for } e \in \mathcal{E}
$$

where $h_{e}$ is the length of the edge $e$, adopting the convention that for $e \in \mathcal{E}$ contributions from outside $\bar{\Omega}$ in the definition of $\sigma_{e}$ are set to zero. Then, assuming that $u \in A$ is such that $\left.u\right|_{\kappa} \in \tilde{H}^{k_{\kappa}+1}(\kappa)$ with $k_{\kappa} \geq 1$, the solution $u_{\mathrm{DG}} \in S^{\mathbf{p}}(\Omega, \mathcal{T}, \mathbf{F}, \mathbf{Q})$ obeys the error bound

$$
\left|\left\|u-\left.u_{\mathrm{DG}}\left|\|_{\mathrm{DG}} \leq C \sum_{\kappa \in \mathcal{T}} \frac{h_{\kappa}^{s_{\kappa}}}{p_{\kappa}^{k_{\kappa}}}\left(\alpha_{\kappa}^{\frac{1}{2}}+\frac{h_{\kappa}}{p_{\kappa}} \gamma_{\kappa}^{\frac{1}{2}}\right)\right| u\right|_{\tilde{H}^{s_{\kappa}+1}(\kappa)} ^{{ }^{2}},\right.\right.
$$

with $1 \leq s_{\kappa} \leq \min \left\{p_{\kappa}, k_{\kappa}\right\}, p_{\kappa} \geq 1, \alpha_{\kappa}$ being the arithmetic average of $\bar{a}_{\tilde{\kappa}}$ on all the elements neighbouring $\kappa$, including $\kappa$ itself, and $C$ is a constant depending on $J_{Q_{\kappa}}$ (the Jacobian of the elemental diffeomorphism), $\tilde{c}_{\kappa}$ (the local shape-regularity constant) for all $\kappa \in \mathcal{T}, \rho$ (the bounded local variation constant), on $\tau$ (the mesh regularity constant), and on $k:=\max _{\kappa \in \mathcal{T}} k_{\kappa}$, but not on $h_{\kappa}$ and $p_{\kappa}$.

Moreover, if $u \in A$ is such that $\left.u\right|_{\kappa} \in H^{k_{\kappa}+1}(\kappa)$ only with $k_{\kappa} \geq 2, \kappa \in \mathcal{T}$, the solution $u_{\mathrm{DG}} \in S^{\mathbf{p}}(\Omega, \mathcal{T}, \mathbf{F}, \mathbf{Q})$ obeys the following error bound

$$
||\left|u-u_{\mathrm{DG}}\right| \|_{\mathrm{DG}} \leq C \sum_{\kappa \in \mathcal{T}} \frac{h_{\kappa}^{s_{\kappa}}}{p_{\kappa}^{k_{\kappa}}}\left(p_{\kappa}^{\frac{1}{2}} \alpha_{\kappa}^{\frac{1}{2}}+\frac{h_{\kappa}}{p_{\kappa}} \gamma_{\kappa}^{\frac{1}{2}}\right)|u|_{H^{s_{\kappa}+1}(\kappa)},
$$

with $2 \leq s_{\kappa} \leq \min \left\{p_{\kappa}, k_{\kappa}\right\}, p_{\kappa} \geq 2$.

Proof Coercivity of the bilinear form $B(\cdot, \cdot)$ is trivial when $\theta=1$, yielding

$$
\left|\|w \mid\|_{\mathrm{DG}}^{2}=B(w, w), \quad \text { for all } w \in \tilde{H}^{1}(\Omega, \mathcal{T}),\right.
$$

for any $\sigma>0$. When $\theta=-1$ we have to choose $\sigma_{e}:=C_{\sigma}\left\langle\bar{a}_{e} p^{2}\right\rangle_{e} / h_{e}$, for $e \in \mathcal{E}$, with $C_{\sigma}$ large enough, to obtain

$$
C\left(C_{\sigma}\right)\left|\|w \mid\|_{\mathrm{DG}}^{2} \leq B(w, w), \quad \text { for all } w \in \tilde{H}^{1}(\Omega, \mathcal{T})\right.
$$

(we refer to the proof of Theorem 4.1 in [1] for details). We decompose the error $u-u_{\mathrm{DG}}$, where $u$ denotes the analytical solution, as $u-u_{\mathrm{DG}}=\eta+\xi$ where $\eta:=u-\Pi_{\mathbf{p}}^{\mathcal{T}} u, \xi:=\Pi_{\mathbf{p}}^{\mathcal{T}} u-u_{\mathrm{DG}}$, with $\Pi_{p}^{\mathcal{T}}$ defined element-wise by

$$
\left.\left(\Pi_{\mathbf{p}}^{\mathcal{T}} u\right)\right|_{\kappa}:=\pi_{p_{\kappa}}^{h}\left(\left.u\right|_{\kappa}\right)
$$

with $\mathbf{p}$ being the polynomial degree distribution, and $\pi_{p_{\kappa}}^{h}$ denoting the $H^{1}$-projection operator on $\kappa$. We then have

$$
\left|\left\|u-u_{\mathrm{DG}}\left|\left\|_{\mathrm{DG}} \leq\left|\left\|\eta \left|\left\|_{\mathrm{DG}}+\mid\right\| \xi \|_{\mathrm{DG}} .\right.\right.\right.\right.\right.\right.\right.
$$


The bound

$$
\begin{aligned}
\|\| \xi \|_{\mathrm{DG}}^{2} \leq & C\left(\int_{\Gamma_{\mathrm{D}}} \sigma|\eta|^{2} \mathrm{~d} s+\int_{\Gamma_{\mathrm{int}}} \sigma[\eta]^{2} \mathrm{~d} s+\sum_{\kappa \in \mathcal{T}}\left(\|\sqrt{a} \nabla \eta\|_{\kappa}^{2}+\|\sqrt{c} \eta\|_{\kappa}^{2}\right)\right. \\
& +\sum_{\kappa \in \mathcal{T}} \bar{a}_{\kappa}\left(\frac{p_{\kappa}^{2}}{h_{\kappa}}\|\eta\|_{\partial \kappa \cap \Gamma_{\mathrm{D}}}^{2}+\frac{\bar{a}_{\kappa}}{\sigma}\|\nabla \eta\|_{\partial \kappa \cap \Gamma_{\mathrm{D}}}^{2}\right) \\
& +\sum_{\kappa \in \mathcal{T}} \bar{a}_{\kappa}\left(\frac{p_{\kappa}^{2}}{h_{\kappa}}\|[\eta]\|_{\partial \kappa \cap \Gamma_{\mathrm{int}}}^{2}+\frac{\bar{a}_{\kappa}}{\sigma}\|\nabla \eta\|_{\partial \kappa \cap \Gamma_{\mathrm{int}}}^{2}\right)
\end{aligned}
$$

from Lemma 4.3 of [5], upon rearrangement yields

$$
\begin{aligned}
\|\xi\|_{\mathrm{DG}}^{2} \leq & C \sum_{\kappa \in \mathcal{T}}\left\{\|\sqrt{a} \nabla \eta\|_{\kappa}^{2}+\|\sqrt{c} \eta\|_{\kappa}^{2}\right. \\
& \left.+\sum_{\substack{e \in \mathcal{E} \\
e \subset \varnothing \kappa}} \bar{a}_{\kappa}\left(\left(\frac{p_{\kappa}^{2}}{h_{\kappa}}+\frac{\sigma_{e}}{\bar{a}_{\kappa}}\right)\|\eta\|_{e}^{2}+\frac{\bar{a}_{\kappa}}{\sigma_{e}}\|\nabla \eta\|_{e}^{2}\right)\right\} .
\end{aligned}
$$

Inserting this into (7.5) and choosing $\sigma$ as in the statement of the theorem, we obtain

$$
\left\|u-u_{\mathrm{DG}}\right\|_{\mathrm{DG}}^{2} \leq C \sum_{\kappa \in \mathcal{T}}\left\{\alpha_{\kappa}\|\nabla \eta\|_{\kappa}^{2}+\gamma_{\kappa}\|\eta\|_{\kappa}^{2}+\sum_{\substack{e \in \mathcal{E} \\ e \subset \complement \delta}} \alpha_{\kappa}\left(\frac{p_{\kappa}^{2}}{h_{\kappa}}\|\eta\|_{e}^{2}+\frac{h_{\kappa}}{p_{\kappa}^{2}}\|\nabla \eta\|_{e}^{2}\right)\right\} .
$$

Making use of the bounds presented in Theorems 7, 8 and 10, and noting that $p_{\kappa}^{-s_{\kappa}} \leq C\left(k_{\kappa}-\right.$ $1)^{k_{\kappa}-1} p_{\kappa}^{-k_{\kappa}}$, we deduce the required bounds.

We have followed a similar approach as in the proof of Theorem 4.5 in [5]. The difference lies in the use of the $H^{1}$-projection described in Section 6 instead of the Babuška-Suri projection operator used therein. Then, upon exploiting the additional regularity of the solution $u$ belonging to the augmented Sobolev space $\tilde{H}^{k_{\kappa}+1}(\kappa)$, we can bound optimally the terms on the right-hand side of (7.6). The crucial improvement lies in the fact that the $H^{1}$-projection operator satisfies the bound (6.26) which admits enhanced convergence in $p$ by half an order of $p$ (cf. Remark 9). This additional order of $p$ "balances" the "bad" factor of the form $p_{\kappa}^{2} / h_{\kappa}$ multiplying $\|\eta\|_{\partial \kappa_{i}}$ in (7.6), which result from the use of an inverse inequality.

Note, however, that the error bound of the $h p$-DGFEM in the energy norm using the $H^{1}$-projection, when the solution belongs element-wise to a standard Sobolev space, continues to exhibit the same mild $p$-suboptimality as the ones in [5]. Therefore, the crucial development here, that results in a fully $h p$-optimal bound, is the use of augmented Sobolev spaces.

To the best of our knowledge, there is no (numerical or analytical) example in the literature which demonstrates $p$-suboptimal convergence for the $h p$-DGFEM for solutions in standard Sobolev spaces. Hence, it remains an open question whether the $p$-optimality of $h p$-DGFEM for solutions in standard Sobolev spaces is a real phenomenon whose proof is lacking, or the method is indeed suboptimal in $p$ by half an order of $p$ and $p$-optimality can be recovered only by assuming additional regularity, as was done here through the use of augmented Sobolev spaces. 


\section{References}

[1] Arnold, D. N. An interior penalty finite element method with discontinuous elements. SIAM J. Numer. Anal. 19 (1982), 742-760.

[2] Cockburn, B., Karniadakis, G. E., and Shu, C.-W. The development of discontinuous Galerkin methods. In Discontinuous Galerkin methods (Newport, RI, 1999), vol. 11 of Lect. Notes Comput. Sci. Eng. Springer, Berlin, 2000, pp. 3-50.

[3] Georgoulis, E. H. Discontinuous Galerkin methods on shape-regular and anisotropic meshes. D.Phil. Thesis, University of Oxford (2003).

[4] Houston, P., Schwab, C., And Süli, E. Stabilized $h p$-finite element methods for first-order hyperbolic problems. SIAM J. Numer. Anal. 37, 5 (2000), 1618-1643 (electronic).

[5] Houston, P., Schwab, C., And Süli, E. Discontinuous $h p$-finite element methods for advection-diffusion-reaction problems. SIAM J. Numer. Anal. 39, 6 (2002), 2133-2163 (electronic).

[6] Nitsche, J. Über ein Variationsprinzip zur Lösung von Dirichlet Problemen bei Verwendung von Teilräumen, die keinen Randbedingungen unterworfen sind. Abh. Math. Sem. Uni. Hamburg 36 (1971), 9-15.

[7] RÅde, L., And Westergren, B. Mathematics handbook for science and engineering, fourth ed. Springer-Verlag, Berlin, 1999.

[8] Rivière, B., Wheeler, M. F., and Girault, V. Improved energy estimates for interior penalty, constrained and discontinuous Galerkin methods for elliptic problems. I. Comput. Geosci. 3, 3-4 (1999), 337-360 (2000).

[9] SchwaB, C. p- and hp- finite element methods: Theory and applications in solid and fluid mechanics. Oxford University Press: Numerical mathematics and scientific computation, 1998.

[10] Wheeler, M. F. An elliptic collocation-finite element method with interior penalties. SIAM J. Numer. Anal. 15, 1 (1978), 152-161.

[11] Wloka, J. Partial differential equations. Cambridge University Press, Cambridge, 1987. Translated from the German by C. B. Thomas and M. J. Thomas. 